Bull. Korean Math. Soc. 46 (2009), No. 2, pp. 373-385

DOI 10.4134/BKMS.2009.46.2.373

\title{
BOOLEAN REGULAR MATRICES AND THEIR STRONGLY PRESERVERS
}

\author{
Seok-Zun Song, Kyung-Tae Kang, and Mun-Hwan Kang \\ ABSTRACT. An $m \times n$ Boolean matrix $A$ is called regular if there exists an \\ $n \times m$ Boolean matrix $X$ such that $A X A=A$. We have characterizations \\ of Boolean regular matrices. We also determine the linear operators that \\ strongly preserve Boolean regular matrices.
}

\section{Introduction}

The Boolean algebra [3] is the set $\mathbb{B}=\{0,1\}$ equipped with two binary operations, addition, + , and multiplication, $\cdot \cdot$, defined as follows:

$$
\begin{array}{cc}
0+0=0 & 0 \cdot 0=0 \\
0+1=1+0=1 & 0 \cdot 1=1 \cdot 0=0 \\
1+1=1 & 1 \cdot 1=1 .
\end{array}
$$

For all $a, b \in \mathbb{B}$, we suppress the dot of $a \cdot b$ and simply write $a b$. Let $\mathbb{M}_{m, n}(\mathbb{B})$ denote the set of all $m \times n$ Boolean matrices with entries in the Boolean algebra $\mathbb{B}$. The usual definitions for addition and multiplication of matrices over fields are applied to Boolean matrices as well. If $m=n$, we use the notation $\mathbb{M}_{n}(\mathbb{B})$ instead of $\mathbb{M}_{m, n}(\mathbb{B})$.

Boolean matrices play an important role in linear algebra, combinatorics, graph theory and network theory. And many problems in the theory of nonnegative matrices depend only on the distribution of nonzero entries. In such cases the relevant property of each entry is whether it is zero or nonzero, and the problem can be often simplified by substituting for the given matrix the Boolean (0,1)-matrix.

Several authors characterized those linear operators on $\mathbb{M}_{m, n}(\mathbb{B})$ that (strongly) preserve various properties and functions defined on $\mathbb{M}_{m, n}(\mathbb{B})$ ([1], [9], $[10])$.

Received June 12, 2008

2000 Mathematics Subject Classification. 15A04, 15A09.

Key words and phrases. Boolean algebra, generalized inverse of a matrix, regular matrix, $(U, V)$-operator.

This work was supported by the Korea Research Foundation Grant funded by the Korean Government (MOEHRD, Basic Research Promotion Fund) (KRF-2007-313-C00003). 
In this paper, we study some properties of Boolean regular matrices. We also determine the linear operators on $\mathbb{M}_{m, n}(\mathbb{B})$ that strongly preserve Boolean regular matrices.

\section{Properties of regularity and singularity of Boolean matrices}

The matrix $I_{n}$ is the $n \times n$ identity matrix, $J_{m, n}$ is the $m \times n$ matrix of all ones, $O_{m, n}$ is the $m \times n$ zero matrix. We will suppress the subscripts on these matrices when the orders are evident from the context. For any matrix $A \in \mathbb{M}_{m, n}(\mathbb{B}), A^{T}$ is denoted by the transpose of $A$. A matrix in $\mathbb{M}_{m, n}(\mathbb{B})$ with only one nonzero entry is called a cell. If the nonzero entry occurs in the $i^{t h}$ row and the $j^{\text {th }}$ column, we denote this cell by $E_{i, j}$.

Definition. A matrix in $\mathbb{M}_{m, n}(\mathbb{B})$ is called an $i^{\text {th }}$ row matrix, denoted by $R_{i}$, if $R_{i}=\sum_{j=1}^{n} E_{i, j}$ for some $i \in\{1, \ldots, m\}$. Similarly, a matrix in $\mathbb{M}_{m, n}(\mathbb{B})$ is called a $j^{\text {th }}$ column matrix, denoted by $C_{j}$, if $C_{j}=\sum_{i=1}^{m} E_{i, j}$ for some $j \in\{1, \ldots, n\}$. A line matrix is an $i^{\text {th }}$ row matrix or a $j^{\text {th }}$ column matrix.

Let $A=\left[a_{i, j}\right]$ be any matrix in $\mathbb{M}_{m, n}(\mathbb{B})$. Then $A$ can be written uniquely as $\sum_{i=1}^{m} \sum_{j=1}^{n} a_{i, j} E_{i, j}$, which is called the canonical form of $A$. If $a_{i, j}=1$ for some $i$ and $j$, then we say that the cell $E_{i, j}$ is in the matrix $A$. Since $a_{i, j} \in\{0,1\}$, the canonical form of $A$ shows that $A$ is a sum of cells.

For $A=\left[a_{i, j}\right], B=\left[b_{i, j}\right] \in \mathbb{M}_{m, n}(\mathbb{B})$, we say that $B$ dominates $A$ (written $B \geq A$ or $A \leq B)$ if $b_{i, j}=0$ implies $a_{i, j}=0$ for all $i$ and $j$. This provides a reflexive and transitive relation on $\mathbb{M}_{m, n}(\mathbb{B})$.

Definition. Cells $E_{1}, E_{2}, \ldots, E_{k}$ are called collinear if $\sum_{i=1}^{k} E_{i} \leq L$ for some line matrix $L$.

Definition. A matrix $A \in \mathbb{M}_{n}(\mathbb{B})$ is said to be invertible if there exists a matrix $B \in \mathbb{M}_{n}(\mathbb{B})$ such that $A B=B A=I_{n}$.

In 1952 , Luce $([4])$ showed that a matrix $A \in \mathbb{M}_{n}(\mathbb{B})$ possesses a two-sided inverse if and only if $A$ is an orthogonal matrix in the sense that $A A^{T}=I_{n}$, and that, in this case, $A^{T}$ is a two-sided inverse of $A$. In 1963, Rutherford ([8]) showed that if a matrix $A \in \mathbb{M}_{n}(\mathbb{B})$ possesses a one-sided inverse, then the inverse is also a two-sided inverse. Furthermore such an inverse, if it exists, is unique and is $A^{T}$. Also, it is well known that the $n \times n$ permutation matrices are the only $n \times n$ invertible Boolean matrices.

The notion of generalized inverse of an arbitrary matrix apparently originated in the work of Moore ([5]), and the generalized inverses have applications in network and switching theory and information theory $([2])$.

Definition. Let $A$ be a matrix in $\mathbb{M}_{m, n}(\mathbb{B})$. Consider a matrix $X \in \mathbb{M}_{n, m}(\mathbb{B})$ in the equation

$$
A X A=A
$$


If (2.1) has a solution $X$, then $X$ is called a generalized inverse of $A$. Furthermore $A$ is called regular if there exists a solution of (2.1); Otherwise, $A$ is called singular.

Clearly $J_{m, n}$ and $O_{m, n}$ are regular in $\mathbb{M}_{m, n}(\mathbb{B})$ because $J_{m, n} J_{n, m} J_{m, n}=$ $J_{m, n}$ and $O_{m, n} O_{n, m} O_{m, n}=O_{m, n}$. In general, a solution of (2.1), although it exists, is not necessarily unique. For example, consider a matrix $A=\left[\begin{array}{ll}1 & 0 \\ 0 & 0\end{array}\right] \in$ $\mathbb{M}_{2}(\mathbb{B})$. Then we can easily show that $X=\left[\begin{array}{ll}1 & a \\ b & c\end{array}\right] \in \mathbb{M}_{2}(\mathbb{B})$ are generalized inverses of $A$ for all $a, b, c \in \mathbb{B}$.

The equation (2.1) has been studied by several authors $([5,6,7])$. Plemmons ([6]) published algorithms for computing generalized inverses of Boolean matrices under certain conditions. Also Rao and Rao ([7]) had characterizations of regular matrices in $\mathbb{M}_{m, n}(\mathbb{B})$.

Proposition 2.1. Let $A$ be a matrix in $\mathbb{M}_{m, n}(\mathbb{B})$. If $U \in \mathbb{M}_{m}(\mathbb{B})$ and $V \in$ $\mathbb{M}_{n}(\mathbb{B})$ are invertible, then the following are equivalent:

(1) $A$ is regular in $\mathbb{M}_{m, n}(\mathbb{B})$;

(2) $U A V$ is regular in $\mathbb{M}_{m, n}(\mathbb{B})$;

(3) $A^{T}$ is regular in $\mathbb{M}_{n, m}(\mathbb{B})$;

(4) $U A^{T} V$ is regular in $\mathbb{M}_{n}(\mathbb{B})$ (if $m=n$ ).

Proof. It is obvious.

Also we can easily show that

$$
A \text { is regular if and only if }\left[\begin{array}{cc}
A & O \\
O & B
\end{array}\right] \text { is regular }
$$

for all matrices $A \in \mathbb{M}_{m, n}(\mathbb{B})$ and for all regular matrices $B \in \mathbb{M}_{p, q}(\mathbb{B})$. In particular, all idempotent matrices in $\mathbb{M}_{n}(\mathbb{B})$ are regular.

Definition. The Boolean rank $([1]), b(A)$, of a nonzero $m \times n$ Boolean matrix $A$ is defined as the least integer $k$ for which there exist $m \times k$ and $k \times n$ Boolean matrices $B$ and $C$ with $A=B C$. The Boolean rank of a zero matrix is zero.

We can easily obtain that

$$
0 \leq b(A) \leq \min \{m, n\} \quad \text { and } \quad b(A B) \leq \min \{b(A), b(B)\}
$$

for all $A \in \mathbb{M}_{m, n}(\mathbb{B})$ and for all $B \in \mathbb{M}_{n, q}(\mathbb{B})$.

Let $A=\left[\begin{array}{llll}\mathbf{a}_{1} & \mathbf{a}_{2} & \cdots & \mathbf{a}_{n}\end{array}\right]$ be a matrix in $\mathbb{M}_{m, n}(\mathbb{B})$, where $\mathbf{a}_{j}$ is the $j^{\text {th }}$ column of $A$ for all $j=1, \ldots, n$. Then the column space of $A$ is the set $\left\{\sum_{j=1}^{n} \alpha_{j} \mathbf{a}_{j} \mid \alpha_{j} \in \mathbb{B}\right\}$, and denoted by $\langle A\rangle ;$ the row space of $A$ is $\left\langle A^{T}\right\rangle$.

Definition. Let $A$ be a matrix in $\mathbb{M}_{m, n}(\mathbb{B})$ with $b(A)=k$. Then $A$ is said to be space decomposable if there exist matrices $B \in \mathbb{M}_{m, k}(\mathbb{B})$ and $C \in \mathbb{M}_{k, n}(\mathbb{B})$ such that $A=B C,\langle A\rangle=\langle B\rangle$ and $\left\langle A^{T}\right\rangle=\left\langle C^{T}\right\rangle$.

Theorem $2.2([7])$. A is regular in $\mathbb{M}_{m, n}(\mathbb{B})$ if and only if $A$ is space decomposable. 
Proposition 2.3. If $A$ is a matrix in $\mathbb{M}_{m, n}(\mathbb{B})$ with $b(A) \leq 2$, then $A$ is regular.

Proof. If $b(A)=0$, then $A=O$ is clearly regular. If $b(A)=1$, then there exist permutation matrices $P$ and $Q$ such that $P A Q=\left[\begin{array}{ll}J & O \\ O & O\end{array}\right]$, and hence $P A Q$ is regular by (2.2). It follows from Proposition 2.1 that $A$ is regular.

Suppose that $b(A)=2$. Then there exist $m \times 2$ matrix $B=\left[\begin{array}{ll}\mathbf{b}_{1} & \mathbf{b}_{2}\end{array}\right]$ and $2 \times n$ matrix $C=\left[\begin{array}{ll}\mathbf{c}_{1} & \mathbf{c}_{2}\end{array}\right]^{T}$ such that $A=B C$, where $\mathbf{b}_{1}$ and $\mathbf{b}_{2}$ are distinct nonzero columns of $B$, and $\mathbf{c}_{1}$ and $\mathbf{c}_{2}$ are distinct nonzero columns of $C^{T}$. Then we can easily show that all columns of $A$ are of the forms $\mathbf{0}, \mathbf{b}_{1}, \mathbf{b}_{2}$ and $\mathbf{b}_{1}+\mathbf{b}_{2}$ so that $\langle A\rangle=\langle B\rangle$. Similarly, all columns of $A^{T}$ are of the forms $\mathbf{0}, \mathbf{c}_{1}, \mathbf{c}_{2}$ and $\mathbf{c}_{1}+\mathbf{c}_{2}$ so that $\left\langle A^{T}\right\rangle=\left\langle C^{T}\right\rangle$. Therefore $A$ is space decomposable and hence $A$ is regular by Theorem 2.2 .

The weight of a matrix $A$ in $\mathbb{M}_{m, n}(\mathbb{B})$ is the number of nonzero entries of $A$ and will be denoted by $|A|$. The number of elements in a set $\mathbb{S}$ is also denoted by $|\mathbb{S}|$.

Corollary 2.4. Let $A$ be a matrix in $\mathbb{M}_{m, n}(\mathbb{B})$ with $|A| \leq 4$. Then $A$ is regular.

Proof. By Proposition 2.3, we lose no generality in assuming that $b(A)=3$ or 4. Consider a matrix $B=\left[\begin{array}{ll}A & O \\ O & 0\end{array}\right]$ in $\mathbb{M}_{m+1, n+1}(\mathbb{B})$. Since $|A| \leq 4$ and $b(A)=3$ or 4 , we can easily show that there exist permutation matrices $P \in \mathbb{M}_{m+1}(\mathbb{B})$ and $Q \in \mathbb{M}_{n+1}(\mathbb{B})$ such that $P B Q=\left[\begin{array}{ll}C & O \\ O & O\end{array}\right]$ for some idempotent matrix $C$ in $\mathbb{M}_{4}(\mathbb{B})$ with $|C|=3$ or 4 . By (2.2) and Proposition 2.1, we have that $B$ is regular and hence $A$ is regular by (2.2).

Example 2.5. Consider a matrix $A=\left[\begin{array}{lll}1 & 1 & 0 \\ 0 & 1 & 1 \\ 0 & 0 & 1\end{array}\right]$. Then we can easily show that $b(A)=3$. Now we show that $A$ is not space decomposable. If $A$ is space decomposable, then there exist $3 \times 3$ matrices $B$ and $C$ such that $A=B C$, $\langle A\rangle=\langle B\rangle$ and $\left\langle A^{T}\right\rangle=\left\langle C^{T}\right\rangle$. It follows from $(2.3)$ that $b(B)=b(C)=3$, and hence both $B$ and $C$ have neither a zero row nor a zero column. Also, there exists a permutation matrix $P$ such that $A=D E$, where $D=\left[d_{i, j}\right]=B P$, $E=\left[e_{i, j}\right]=P^{T} C$ and $D \geq I_{3}$. Then we have

$$
\langle A\rangle=\langle B\rangle=\langle B P\rangle=\langle D\rangle
$$

and

$$
\left\langle A^{T}\right\rangle=\left\langle C^{T}\right\rangle=\left\langle C^{T} P\right\rangle=\left\langle E^{T}\right\rangle .
$$

Furthermore we have that

$$
E \text { has neither a zero row nor a zero column }
$$

because $b(E)=b\left(P^{T} C\right)=b(C)=3$. From $A=D E$ with $a_{1,3}=a_{2,1}=a_{3,1}=$ $a_{3,2}=0$, we have $e_{1,3}=e_{2,1}=e_{3,1}=e_{3,2}=0$. It follows from (2.6) that $e_{1,1}=e_{3,3}=1$. Thus, $E=\left[\begin{array}{ccc}1 & e_{1,2} & 0 \\ 0 & e_{2,2} & e_{2,3} \\ 0 & 0 & 1\end{array}\right]$. If $e_{1,2}=0$ or $e_{2,3}=0$, then we have 
$e_{2,2}=1$ by (2.6). Then we have $\left[\begin{array}{l}0 \\ 1 \\ 0\end{array}\right] \in\left\langle E^{T}\right\rangle$, while $\left[\begin{array}{l}0 \\ 1 \\ 0\end{array}\right] \notin\left\langle A^{T}\right\rangle$, a contradiction to $(2.5)$. Thus we may assume that $e_{1,2}=e_{2,3}=1$ so that $E=\left[\begin{array}{ccc}1 & 1 & 0 \\ 0 & e_{2,2} & 1 \\ 0 & 0 & 1\end{array}\right]$. If $e_{2,2}=0$, then $b(E)=2$, a contradiction. Hence $e_{2,2}=1$. It follows from $A=D E$ that $D=\left[\begin{array}{ccc}1 & 0 & 0 \\ 0 & 1 & d_{2,3} \\ 0 & 0 & 1\end{array}\right]$. In this case, $\left[\begin{array}{l}0 \\ 1 \\ 0\end{array}\right] \in\langle D\rangle$, while $\left[\begin{array}{l}0 \\ 1 \\ 0\end{array}\right] \notin\langle A\rangle$, a contradiction to (2.4). Therefore $A$ is not space decomposable.

In the following, we give some properties of Boolean regular matrices.

If $A$ and $B$ are matrices in $\mathbb{M}_{m, n}(\mathbb{B})$, we define $A \backslash B$ to be the matrix $C=\left[c_{i, j}\right]$ in $\mathbb{M}_{m, n}(\mathbb{B})$ such that $c_{i, j}=1$ if and only if $a_{i, j}=1$ and $b_{i, j}=0$.

Define an upper triangular matrix $\Lambda_{n}$ in $\mathbb{M}_{n}(\mathbb{B})$ by

$$
\Lambda_{n}=\left[\lambda_{i, j}\right] \equiv\left(\sum_{i \leq j}^{n} E_{i, j}\right) \backslash E_{1, n}=\left[\begin{array}{ccccc}
1 & 1 & \cdots & 1 & 0 \\
& 1 & \cdots & 1 & 1 \\
& & \ddots & \vdots & \vdots \\
& & & 1 & 1 \\
& & & & 1
\end{array}\right] .
$$

Then the following Lemma shows that $\Lambda_{n}$ is not regular for $n \geq 3$.

Lemma 2.6. $\Lambda_{n}$ is regular in $\mathbb{M}_{n}(\mathbb{B})$ if and only if $n \leq 2$.

Proof. If $n \leq 2$, then $\Lambda_{n}$ is regular by Corollary 2.4 .

Conversely, assume that $\Lambda_{n}$ is regular for some $n \geq 3$. Then there exists a nonzero matrix $B=\left[b_{i, j}\right]$ in $\mathbb{M}_{n}(\mathbb{B})$ such that $\Lambda_{n}=\Lambda_{n} B \Lambda_{n}$. From $0=$ $\lambda_{1, n}=\sum_{i=1}^{n-1} \sum_{j=2}^{n} b_{i, j}$, we obtain that all entries of the second column of $B$ are zero except for the entry $b_{n, 2}$. From $0=\lambda_{2,1}=\sum_{i=2}^{n} b_{i, 1}$, we have that all entries of the first column of $B$ are zero except for $b_{1,1}$. Also, from $0=\lambda_{3,2}=\sum_{i=3}^{n} \sum_{j=1}^{2} b_{i, j}$, we obtain that $b_{n, 2}=0$. If we combine these three results, we conclude that all entries of the first two columns are zero except for $b_{1,1}$. But we have $1=\lambda_{2,2}=\sum_{i=2}^{n} \sum_{j=1}^{2} b_{i, j}=0$, a contradiction. Hence $\Lambda_{n}$ is singular for all $n \geq 3$.

In particular, $\Lambda_{3}=\left[\begin{array}{lll}1 & 1 & 0 \\ 0 & 1 & 1 \\ 0 & 0 & 1\end{array}\right]$ is singular. By Proposition 2.1, we have that the lower triangular matrix $\Lambda_{n}^{T}$ is singular for $n \geq 3$, while $\Lambda_{n}+\Lambda_{n}^{T}$ is regular by Proposition 2.3 because $b\left(\Lambda_{n}+\Lambda_{n}^{T}\right)=2$. Let

$$
\Phi_{m, n}=\left[\begin{array}{cc}
\Lambda_{3} & O \\
O & O
\end{array}\right]
$$

for all $\min \{m, n\} \geq 3$. Then $\Phi_{m, n}$ is singular by (2.2).

Corollary 2.7. Let $E$ and $F$ be distinct cells in $\mathbb{M}_{m, n}(\mathbb{B})$ with $\min \{m, n\} \geq 3$. Then there exists a matrix $A$ in $\mathbb{M}_{m, n}(\mathbb{B})$ such that $|A|=3$ and $A+E+F$ is singular in $\mathbb{M}_{m, n}(\mathbb{B})$. 
Proof. Since $E$ and $F$ are distinct cells, there exist permutation matrices $P$ and $Q$ such that

$$
P(E+F) Q=E_{1,1}+E_{1,2}, \quad E_{1,2}+E_{2,2} \quad \text { or } \quad E_{1,1}+E_{2,2} .
$$

Consider a matrix $A \in \mathbb{M}_{m, n}(\mathbb{B})$ such that

$$
P A Q=E_{2,2}+E_{2,3}+E_{3,3}, \quad E_{1,1}+E_{2,3}+E_{3,3} \quad \text { or } \quad E_{1,2}+E_{2,3}+E_{3,3}
$$

according as $P(E+F) Q=E_{1,1}+E_{1,2}, \quad E_{1,2}+E_{2,2}$ or $E_{1,1}+E_{2,2}$. Then we have that $P(A+E+F) Q=\Phi_{m, n}$ is singular in $\mathbb{M}_{m, n}(\mathbb{B})$. Hence $A+E+F$ is singular in $\mathbb{M}_{m, n}(\mathbb{B})$ by Proposition 2.1 .

Corollary 2.8. Let $A$ be a matrix in $\mathbb{M}_{m, n}(\mathbb{B})$ with $|A|=3$. If $b(A)=2$ or 3 , then there exist cells $E$ and $F$ such that $A+E+F$ is singular.

Proof. Consider the singular matrix $\Phi_{m, n}$ in (2.7). If $b(A)=2$ or 3 , then we can easily show that there exist permutation matrices $U$ and $V$ such that $U A V \leq \Phi_{m, n}$. Let $E^{\prime}$ and $F^{\prime}$ be cells satisfying $U A V+E^{\prime}+F^{\prime}=\Phi_{m, n}$. Then we obtain that

$$
A+U^{T} E^{\prime} V^{T}+U^{T} F^{\prime} V^{T}=U^{T} \Phi_{m, n} V^{T}
$$

is singular by Proposition 2.1. If we let $E=U^{T} E^{\prime} V^{T}$ and $F=U^{T} F^{\prime} V^{T}$, then the result follows.

Theorem 2.9. For $m \geq 3$ and $n \geq 3$, let $A$ be a matrix in $\mathbb{M}_{m, n}(\mathbb{B})$ with $|A|=k$ and $b(A)=k$, where $0 \leq k \leq \min \{m, n\}$. Then $J \backslash A$ is regular if and only if $k \leq 2$.

Proof. If $k \leq 2$, then there exist permutation matrices $P$ and $Q$ such that $P(J \backslash A) Q=J \backslash\left(a E_{1,1}+b E_{2,2}\right)$, where $a, b \in\{0,1\}$, and hence

$$
\begin{gathered}
J \backslash E_{11}=\left[\begin{array}{cc}
1 & 0 \\
1 & 1 \\
\vdots & \vdots \\
1 & 1
\end{array}\right]\left[\begin{array}{llll}
0 & 1 & \cdots & 1 \\
1 & 1 & \cdots & 1
\end{array}\right], \\
J \backslash E_{22}=\left[\begin{array}{cc}
1 & 1 \\
1 & 0 \\
1 & 1 \\
\vdots & \vdots \\
1 & 1
\end{array}\right]\left[\begin{array}{ccccc}
1 & 0 & 1 & \cdots & 1 \\
1 & 1 & 1 & \cdots & 1
\end{array}\right], \\
J \backslash E_{11}+E_{22}=\left[\begin{array}{cc}
1 & 0 \\
0 & 1 \\
1 & 1 \\
\vdots & \vdots \\
1 & 1
\end{array}\right]\left[\begin{array}{lllll}
0 & 1 & 1 & \cdots & 1 \\
1 & 0 & 1 & \cdots & 1
\end{array}\right] .
\end{gathered}
$$


Thus $b(J \backslash A)=b(P(J \backslash A) Q) \leq 2$. Therefore we have $J \backslash A$ is regular by Proposition 2.3.

Conversely, assume that $J \backslash A$ is regular for some $k \geq 3$. It follows from $|A|=k$ and $b(A)=k$ that there exist permutation matrices $U$ and $V$ such that

$$
U\left(J_{m, n} \backslash A\right) V=J \backslash \sum_{t=1}^{k} E_{t, t} .
$$

Let $J \backslash\left(\sum_{t=1}^{k} E_{t, t}\right)=X=\left[x_{i, j}\right]$. By Proposition 2.1, $X$ is regular, and hence there exists a nonzero matrix $B=\left[b_{i, j}\right] \in \mathbb{M}_{n, m}(\mathbb{B})$ such that $X=X B X$. Then the $(t, t)^{t h}$ entry of $X B X$ becomes

$$
\sum_{i \in I} \sum_{j \in J} b_{i, j}
$$

for all $t=1, \ldots, k$, where $I=\{1, \ldots, n\} \backslash\{t\}$ and $J=\{1, \ldots, m\} \backslash\{t\}$. From $x_{1,1}=0$ and (2.8), we have that

$$
b_{i, j}=0 \quad \text { for all } i=2, \ldots, n ; j=2, \ldots, m .
$$

Consider the first row and the first column of $B$. It follows from $x_{2,2}=0$ and (2.8) that

$$
b_{i, 1}=0=b_{1, j} \quad \text { for all } i=1,3,4, \ldots, n ; j=1,3,4, \ldots, m .
$$

Also, from $x_{3,3}=0$, we obtain $b_{1,2}=b_{2,1}=0$, and hence $B=O$ by $(2.9)$ and (2.10). This contradiction shows that $k \leq 2$.

Proposition 2.10. Let $A$ be a matrix in $\mathbb{M}_{m, n}(\mathbb{B})$ with $|A|=5$. If $A$ has a row or a column that has at least 3 nonzero entries, then $A$ is regular.

Proof. Suppose that $A$ has a row or a column that has at least 3 nonzero entries. Then we can easily show that $b(A) \leq 3$. By Proposition 2.3, we may assume that $b(A)=3$. Then $A$ has either a row or a column that has just 3 nonzero entries. Suppose that a row of $A$ has just 3 nonzero entries. Since $b(A)=3$, there exist permutation matrices $U$ and $V$ such that

$$
U A V=E_{1,1}+E_{1,2}+E_{1,3}+E_{2, i}+E_{3, j}
$$

for some $i, j \in\{1, \ldots, n\}$ with $i<j$. If $j \geq 4$, then $U A V$ is regular by Corollary 2.4 and (2.2), and hence $A$ is regular by Proposition 2.1. If $1 \leq i<$ $j \leq 3$, then there exist permutation matrices $U^{\prime}$ and $V^{\prime}$ such that

$$
U^{\prime} U A V V^{\prime}=\left[\begin{array}{ll}
B & O \\
O & O
\end{array}\right]
$$

where $B=\left[\begin{array}{lll}1 & 1 & 1 \\ 0 & 1 & 0 \\ 0 & 0 & 1\end{array}\right]$. We can easily show that $B$ is idempotent in $\mathbb{M}_{3}(\mathbb{B})$, and hence $B$ is regular. It follows from (2.2) and Proposition 2.1 that $A$ is regular.

If a column of $A$ has just 3 nonzero entries, a parallel argument shows that $A$ is regular. 


\section{Linear operators that preserve Boolean regular matrices}

In this section we have characterizations of the linear operators that strongly preserve regular matrices over the Boolean algebra.

Definition. An operator $T$ on $\mathbb{M}_{m, n}(\mathbb{B})$ is said to be

(1) linear if $T(\alpha A+\beta B)=\alpha T(A)+\beta T(B)$ for all $\alpha, \beta \in \mathbb{B}$ and for all $A, B \in \mathbb{M}_{m, n}(\mathbb{B})$

(2) preserve regularity if $T(A)$ is regular whenever $A$ is regular in $\mathbb{M}_{m, n}(\mathbb{B})$.

Example 3.1. Let $A$ be any regular matrix in $\mathbb{M}_{m, n}(\mathbb{B})$. Define an operator $T$ on $\mathbb{M}_{m, n}(\mathbb{B})$ by

$$
T(X)=\left(\sum_{i=1}^{m} \sum_{j=1}^{n} x_{i, j}\right) A
$$

for all $X=\left[x_{i, j}\right] \in \mathbb{M}_{m, n}(\mathbb{B})$. Then we can easily show that $T$ is a linear operator that preserves regularity because $T(X)$ is either $O$ or $A$ for all $X \in$ $\mathbb{M}_{m, n}(\mathbb{B})$. But $T$ does not preserve any singular matrix in $\mathbb{M}_{m, n}(\mathbb{B})$.

Thus, we are interested in a linear operator $T$ on $\mathbb{M}_{m, n}(\mathbb{B})$ such that $T(X)$ is regular if and only if $X$ is regular over $\mathbb{M}_{m, n}(\mathbb{B})$.

Definition. A linear operator $T$ on $\mathbb{M}_{m, n}(\mathbb{B})$ is said to be strongly preserve regularity if $T(A)$ is regular if and only if $A$ is regular in $\mathbb{M}_{m, n}(\mathbb{B})$.

Theorem 3.2. Let $T$ be a linear operator on $\mathbb{M}_{m, n}(\mathbb{B})$, where $\min \{m, n\} \leq 2$. Then $T$ strongly preserves all regular matrices.

Proof. If $\min \{m, n\} \leq 2$, then all matrices in $\mathbb{M}_{m, n}(\mathbb{B})$ are regular by $(2.3)$ and Proposition 2.3. Hence $T(A)$ is always regular for all $A$ in $\mathbb{M}_{m, n}(\mathbb{B})$. Thus the result follows.

Definition. A linear operator $T$ on $\mathbb{M}_{m, n}(\mathbb{B})$ is said to be singular if $T(X)=O$ for some nonzero matrix $X$ in $\mathbb{M}_{m, n}(\mathbb{B})$; Otherwise, $T$ is called nonsingular.

Lemma 3.3. If $T$ is a linear operator on $\mathbb{M}_{m, n}(\mathbb{B})$ that strongly preserves regularity for $m \geq 3$ and $n \geq 3$, then $T$ is nonsingular.

Proof. If $T(X)=O$ for some nonzero matrix $X$ in $\mathbb{M}_{m, n}(\mathbb{B})$, then we have $T(E)=O$ for all cells $E \leq X$. Let $F$ be a cell different from $E$. By Corollary 2.7, there exists a matrix $A$ with $|A|=3$ such that $A+E+F$ is singular, while $A+F$ is regular by Corollary 2.4. Nevertheless, $T(A+E+F)=T(A+F)$, a contradiction to the fact that $T$ strongly preserves regularity. Hence $T(X) \neq O$ for all nonzero matrix $X$ in $\mathbb{M}_{m, n}(\mathbb{B})$. Therefore $T$ is nonsingular.

For any $i \in\{1,2, \ldots, m n\}$, let $S_{i}$ denote a sum of arbitrary distinct $i$ cells in $\mathbb{M}_{m, n}(\mathbb{B})$ with $\left|S_{i}\right|=i$. Hereafter, we let $\min \{m, n\}=\alpha$ and $\max \{m, n\}=\beta$. 
Proposition 3.4. Let $T$ be a linear operator on $\mathbb{M}_{m, n}(\mathbb{B})$ that strongly preserves regularity, where $\min \{m, n\}=\alpha \geq 3$. Then we have

$$
\left|T\left(S_{i}\right)\right| \leq 2 \alpha+i
$$

for all $S_{i} \in \mathbb{M}_{m, n}(\mathbb{B})$, where $i \in\{1,2, \ldots, \alpha(\beta-2)\}$.

Proof. We lose no generality in assuming that $\alpha=m$ and $\beta=n$. Thus we will show that $\left|T\left(S_{i}\right)\right| \leq 2 m+i$ for all $S_{i} \in \mathbb{M}_{m, n}(\mathbb{B})$, where $i \in\{1,2, \ldots, m(n-2)\}$.

If $i=m(n-2)$, then clearly $\left|T\left(S_{i}\right)\right| \leq m n=2 m+i$. For arbitrary $i \in$ $\{1,2, \ldots, m(n-2)-1\}$, suppose that $\left|T\left(S_{i}\right)\right| \geq 2 m+i+1$ for some $S_{i} \in$ $\mathbb{M}_{m, n}(\mathbb{B})$. Then $J \backslash T\left(S_{i}\right)$ dominates at most $m n-(2 m+i+1)$ cells. Thus we have $\left|T(J) \backslash T\left(S_{i}\right)\right| \leq m n-(2 m+i+1)$. Now for each cell $G$ with $G \leq$ $T(J) \backslash T\left(S_{i}\right)$, let $H$ be a cell such that $G \leq T(H)$, and let $X$ be the sum of all such cells $H$. Then we have

$$
|X| \leq\left|T(J) \backslash T\left(S_{i}\right)\right| \leq m n-(2 m+i+1) .
$$

Now we claim that $T(J)=T\left(S_{i}\right)+T(X)$. It suffices to show $T(J) \leq T\left(S_{i}\right)+$ $T(X)$. Let $G$ be any cell such that $G \leq T(J)$. If $G \leq T\left(S_{i}\right)$, then we are done. If $G \not \leq T\left(S_{i}\right)$, then there exists a cell $H$ with $H \leq X$ such that $G \leq T(H)$ by the construction of $X$. Thus, $G \leq T(H) \leq T(X)$. Therefore we have $T(J) \leq T\left(S_{i}\right)+T(X)$, and hence $T(J)=T\left(S_{i}\right)+T(X)=T\left(S_{i}+X\right)$.

Since $\left|X+S_{i}\right| \leq m n-(2 m+1)$, there exist distinct cells $F_{1}, F_{2}, F_{3}$ such that they are not dominated by $X+S_{i}$ and $b\left(\sum_{j=1}^{3} F_{j}\right)=3$. It follows from $T(J)=T\left(X+S_{i}\right)$ and $X+S_{i} \leq J \backslash \sum_{j=1}^{3} F_{j}$ that

$$
T(J)=T\left(X+S_{i}\right) \leq T\left(J \backslash \sum_{j=1}^{3} F_{j}\right) \leq T(J),
$$

and hence $T(J)=T\left(J \backslash \sum_{j=1}^{3} F_{j}\right)$, a contradiction to the fact that $T$ strongly preserves regularity because $J$ is regular, while $J \backslash \sum_{j=1}^{3} F_{j}$ is not regular by Theorem 2.9. Therefore we have $\left|T\left(S_{i}\right)\right| \leq 2 m+i$ for all $S_{i}$. We conclude that $\left|T\left(S_{i}\right)\right| \leq 2 m+i$ for all $i=1,2, \ldots, m(n-2)$.

The next Lemma will be important in order to show that if $E$ is any cell in $\mathbb{M}_{m, n}(\mathbb{B})$ with $\min \{m, n\} \geq 3$, then $T(E)$ is also a cell for any linear operator on $\mathbb{M}_{m, n}(\mathbb{B})$ that strongly preserves regularity.

Lemma 3.5. Let $\min \{m, n\}=\alpha \geq 3$ and $T$ be a linear operator on $\mathbb{M}_{m, n}(\mathbb{B})$ that strongly preserves regularity. Then for any $h \in\{0,1,2, \ldots, 2 \alpha\}$, we have

$$
\left|T\left(S_{i}\right)\right| \leq 2 \alpha+i-h
$$

for all $S_{i} \in \mathbb{M}_{m, n}(\mathbb{B})$, where $i \in\{1,2, \ldots, 2 \alpha-h+1\}$.

Proof. Without loss of generality, we assume that $\alpha=m$. Thus we will show that if $h \in\{0,1,2, \ldots, 2 m\}$, then we have $\left|T\left(S_{i}\right)\right| \leq 2 m+i-h$ for all $S_{i} \in$ $\mathbb{M}_{m, n}(\mathbb{B})$, where $i \in\{1,2, \ldots, 2 m-h+1\}$. 
The proof proceeds by induction on $h$. It follows from Proposition 3.4 that $\left|T\left(S_{i}\right)\right| \leq 2 m+i$ for all $S_{i} \in \mathbb{M}_{m, n}(\mathbb{B})$, where $i \in\{1,2, \ldots, 2 m+1\}$. Thus if $h=$ 0 , the result is obvious. Next, we assume that for some $h \in\{0,1,2, \ldots, 2 m-1\}$, the argument is true. That is, we have

$$
\left|T\left(S_{i}\right)\right| \leq 2 m+i-h
$$

for all $S_{i} \in \mathbb{M}_{m, n}(\mathbb{B})$, where $i \in\{1,2, \ldots, 2 m-h+1\}$. Now we will show that $\left|T\left(S_{i}\right)\right| \leq 2 m+i-h-1$ for all $S_{i} \in \mathbb{M}_{m, n}(\mathbb{B})$, where $i \in\{1,2, \ldots, 2 m-h\}$. For arbitrary $i \in\{1,2, \ldots, 2 m-h\}$, suppose that $\left|T\left(S_{i}\right)\right| \geq 2 m+i-h$ for some $S_{i} \in \mathbb{M}_{m, n}(\mathbb{B})$. By (3.1), we have

$$
\left|T\left(S_{i}\right)\right|=2 m+i-h \quad \text { and } \quad\left|T\left(S_{i}+F\right)\right|=2 m+i-h \text { or }(2 m+i-h)+1
$$

for all cells $F$ with $F \not \leq S_{i}$. If $\left|T\left(S_{i}+F_{1}\right)\right|=2 m+i-h$ for some cell $F_{1}$ with $F_{1} \not \leq S_{i}$, then we have $T\left(S_{i}+F_{1}\right)=T\left(S_{i}\right)$. Let $F_{2}$ and $F_{3}$ be distinct cells different from $F_{1}$ such that they are not dominated by $S_{i}$ and $b\left(\sum_{j=1}^{3} F_{j}\right)=3$. Then we can select the matrix $Y \in \mathbb{M}_{m, n}(\mathbb{B})$ such that $S_{i}+Y=J \backslash \sum_{j=1}^{3} F_{j}$, and hence $S_{i}+Y+F_{1}=J \backslash\left(F_{2}+F_{3}\right)$. It follows from $T\left(S_{i}+F_{1}\right)=T\left(S_{i}\right)$ that $T\left(S_{i}+F_{1}\right)+T(Y)=T\left(S_{i}\right)+T(Y)$, equivalently

$$
T\left(J \backslash\left(F_{2}+F_{3}\right)\right)=T\left(J \backslash \sum_{j=1}^{3} F_{j}\right),
$$

a contradiction because $J \backslash \sum_{j=1}^{3} F_{j}$ is singular, while $J \backslash\left(F_{2}+F_{3}\right)$ is regular by Theorem 2.9. Thus we may assume that $\left|T\left(S_{i}+F\right)\right|=(2 m+i-h)+1$ for all cells $F$ with $F \not \leq S_{i}$. This means that for any cell $F$ with $F \not \leq S_{i}$, there exists only one cell $C_{F}$ such that

$$
C_{F} \not \leq T\left(S_{i}\right), \quad C_{F} \leq T(F) \quad \text { and } \quad T\left(S_{i}+F\right)=T\left(S_{i}\right)+C_{F}
$$

because $\left|T\left(S_{i}\right)\right|=2 m+i-h$. Let $\mathbb{E}_{m, n}$ be the set of all cells in $\mathbb{M}_{m, n}(\mathbb{B})$ and let

$$
\Omega=\left\{C_{F} \mid F \in \mathbb{E}_{m, n} \text { and } F \not \leq S_{i}\right\} .
$$

Suppose that $C_{H} \neq C_{F}$ for all distinct cells $F$ and $H$ that are not dominated by $S_{i}$. Then we have $|\Omega|=m n-i$. Since $C_{F} \not \leq T\left(S_{i}\right)$ for any cell $F$ with $F \not \leq S_{i}$, we have $|\Omega| \leq m n-(2 m+i-h)$ because $\left|T\left(S_{i}\right)\right|=2 m+i-h$. This is impossible. Hence $C_{H}=C_{F}$ for some distinct cells $F$ and $H$ that are not dominated by $S_{i}$. It follows from (3.2) that

(3.3) $T\left(S_{i}+F+H\right)=T\left(S_{i}+F\right)+T\left(S_{i}+H\right)=T\left(S_{i}\right)+C_{F}=T\left(S_{i}+F\right)$.

Let $H_{1}$ and $H_{2}$ be distinct cells different from $H$ such that they are not dominated by $S_{i}+F$ and $b\left(H+H_{1}+H_{2}\right)=3$. Let $Y^{\prime}$ be the matrix such that $S_{i}+F+Y^{\prime}=J \backslash\left(H+H_{1}+H_{2}\right)$. Then we have $S_{i}+F+H+Y^{\prime}=J \backslash\left(H_{1}+H_{2}\right)$. It follows from (3.3) that

$$
T\left(J \backslash\left(H_{1}+H_{2}\right)\right)=T\left(J \backslash\left(H+H_{1}+H_{2}\right)\right),
$$


a contradiction because $J \backslash\left(H_{1}+H_{2}\right)$ is regular, while $J \backslash\left(H+H_{1}+H_{2}\right)$ is singular by Theorem 2.9. Consequently, we have $\left|T\left(S_{i}\right)\right| \leq 2 m+i-h$ for all $S_{i} \in \mathbb{M}_{m, n}(\mathbb{B})$, where $i \in\{1,2, \ldots, 2 m-h\}$. Hence the result follows.

Corollary 3.6. Let $T$ be a linear operator on $\mathbb{M}_{m, n}(\mathbb{B})$ that strongly preserves regularity, where $\min \{m, n\} \geq 3$. Then $T(E)$ is a cell for all cells $E$ in $\mathbb{M}_{m, n}(\mathbb{B})$.

Proof. Let $h=2 m$ in Lemma 3.5. Then we have $\left|T\left(S_{1}\right)\right| \leq 1$ for all $S_{1} \in$ $\mathbb{M}_{m, n}(\mathbb{B})$. It follows from Lemma 3.3 that $\left|T\left(S_{1}\right)\right|=1$ for all $S_{1} \in \mathbb{M}_{m, n}(\mathbb{B})$, equivalently $|T(E)|=1$ for any cell $E$ in $\mathbb{M}_{m, n}(\mathbb{B})$. Therefore we have that $T(E)$ is a cell for any cell $E$ in $\mathbb{M}_{m, n}(\mathbb{B})$.

As shown in Theorem 3.2 , if $T$ is a linear operator on $\mathbb{M}_{m, n}(\mathbb{B})$ with $\min \{m$, $n\} \leq 2$, then $T$ (strongly) preserves regularity because all matrices in $\mathbb{M}_{m, n}(\mathbb{B})$ are regular by Proposition 2.3.

If $\min \{m, n\} \geq 3$, there exists a linear operator on $\mathbb{M}_{m, n}(\mathbb{B})$ such that $T$ preserves regularity, while $T$ does not strongly preserve regularity, see Example 3.1.

The next Lemmas are necessary to prove the main theorem. In the followings, unless otherwise stated, we assume that $T$ is a linear operator on $\mathbb{M}_{m, n}(\mathbb{B})$ that strongly preserve regularity for $\min \{m, n\} \geq 3$.

Lemma 3.7. $T$ is bijective on the set of cells.

Proof. By Corollary 3.6, we suffice to show that $T(E) \neq T(F)$ for all distinct cells $E$ and $F$ in $\mathbb{M}_{m, n}(\mathbb{B})$. Suppose that $T(E)=T(F)$ for some distinct cells $E$ and $F$. Then we have $T(E+F)=T(E)$. By Corollary 2.7, there exists a matrix $A$ in $\mathbb{M}_{m, n}(\mathbb{B})$ with $|A|=3$ such that $A+E+F$ is singular. Since $T(E+F)=T(E)$, we have

$$
T(A+E+F)=T(A+E),
$$

a contradiction to the fact that $T$ strongly preserves regularity because $A+E$ is regular by Corollary 2.4. Therefore $T$ is bijective on the set of cells.

Let $A \in \mathbb{M}_{m, n}(\mathbb{B})$ be a nonzero matrix dominated by a line matrix. Then we have $b(A)=1$. If $|A|=s$, then we say that $A$ is a $s$-star matrix. Therefore all $s$-star matrices are regular by Proposition 2.3.

Lemma 3.8. $T$ preserves all 3-star matrices.

Proof. Suppose that $T$ does not preserve a 3 -star matrix $A$ in $\mathbb{M}_{m, n}(\mathbb{B})$. Then we have that $b(T(A))=2$ or 3 . By Corollary 2.8, there exist cells $E$ and $F$ such that $T(A)+E+F$ is singular. By Lemma 3.7, we can write $E=T\left(H_{1}\right)$ and $F=T\left(H_{2}\right)$ for some cells $H_{1}$ and $H_{2}$. Thus we have

$$
T(A)+E+F=T\left(A+H_{1}+H_{2}\right) .
$$

But $A+H_{1}+H_{2}$ is regular by Proposition 2.10. This contradicts to the fact that $T$ strongly preserves regularity. Hence $T$ preserves all 3 -star matrices. 
Corollary 3.9. T preserves all line matrices.

Proof. Suppose that $T$ does not preserve a line matrix $A$ in $\mathbb{M}_{m, n}(\mathbb{B})$. Then there exist two cells $E$ and $F$ dominated by $A$ such that two cells $T(E)$ and $T(F)$ are not collinear. Let $G$ be a cell such that $E+F+G$ is a 3 -star matrix. By Lemma 3.8, $T(E+F+G)$ is a 3-star matrix, and hence $b(T(E+F+G))=1$. Thus, the three cells $T(E), T(F)$ and $T(G)$ are collinear. This contradicts to the fact that the two cells $T(E)$ and $T(F)$ are not collinear. Therefore $T$ preserves all line matrices.

We say that a linear operator $T$ on $\mathbb{M}_{m, n}(\mathbb{B})$ is a $(U, V)$-operator if there exist invertible matrices $U \in \mathbb{M}_{m}(\mathbb{B})$ and $V \in \mathbb{M}_{n}(\mathbb{B})$ such that either

$$
\begin{gathered}
T(X)=U X V \text { for all } X \in \mathbb{M}_{m, n}(\mathbb{B}), \text { or } \\
m=n \text { and } T(X)=U X^{T} V \text { for all } X \in \mathbb{M}_{m, n}(\mathbb{B}) .
\end{gathered}
$$

We remind that the $n \times n$ permutation matrices are the only $n \times n$ invertible Boolean matrices.

Theorem 3.10. Let $T$ be a linear operator on $\mathbb{M}_{m, n}(\mathbb{B})$ with $\min \{m, n\} \geq 3$. Then $T$ strongly preserves regularity if and only if $T$ is a $(U, V)$-operator.

Proof. The sufficiency follows from Proposition 2.1. To prove the necessity, assume that $T$ strongly preserves regularity. Then $T$ is bijective on the set of cells by Lemma 3.7 and $T$ preserves all line matrices by Corollary 3.9. Since no combination of $s$ row matrices and $t$ column matrices can dominate $J_{m, n}$ where $s+t=\min \{m, n\}$ unless $s=0$ or $t=0$, we have that either

(1) the image of each row matrix is a row matrix and the image of each column matrix is a column matrix, or

(2) the image of each row matrix is a column matrix and the image of each column matrix is a row matrix.

If (1) holds, then there exist permutations $\sigma$ and $\tau$ of $\{1, \ldots, m\}$ and $\{1, \ldots$, $n\}$, respectively such that $T\left(R_{i}\right)=R_{\sigma(i)}$ and $T\left(C_{j}\right)=C_{\tau(j)}$ for all $i=1, \ldots, m$ and $j=1, \ldots, n$. Let $U \in \mathbb{M}_{m}(\mathbb{B})$ and $V \in \mathbb{M}_{n}(\mathbb{B})$ be permutation (i.e., invertible) matrices corresponding to $\sigma$ and $\tau$, respectively. Then we have

$$
T\left(E_{i, j}\right)=E_{\sigma(i), \tau(j)}=U E_{i, j} V
$$

for all cells $E_{i, j}$ in $\mathbb{M}_{m, n}(\mathbb{B})$. Let $X=\sum_{i=1}^{m} \sum_{j=1}^{n} x_{i, j} E_{i, j}$ be any matrix in $\mathbb{M}_{m, n}(\mathbb{B})$. By the action of $T$ on the cells, we have that $T(X)=U X V$. If (2) holds, then $m=n$ and a parallel argument shows that there exist invertible matrices $U$ and $V$ in $\mathbb{M}_{n}(\mathbb{B})$ such that $T(X)=U X^{T} V$ for all $X$ in $\mathbb{M}_{n}(\mathbb{B})$. Therefore $T$ is a $(U, V)$-operator.

Thus, as shown in Theorems 3.2 and 3.10, we have characterizations of the linear operators that strongly preserve Boolean regular matrices. 


\section{References}

[1] L. B. Beasley and N. J. Pullman, Boolean-rank-preserving operators and Boolean-rank-1 spaces, Linear Algebra Appl. 59 (1984), 55-77.

[2] J. Dénes, Transformations and transformation semigroups, Seminar Report, University of Wisconsin, Madison, Wisconsin, 1976.

[3] K. H. Kim, Boolean Matrix Theory and Applications, Pure and Applied Mathematics, Vol. 70, Marcel Dekker, New York, 1982.

[4] R. D. Luce, A note on Boolean matrix theory, Proc. Amer. Math. Soc. 3 (1952), 382-388.

[5] E. H. Moore, General analysis, Part I, Mem. of Amer. Phil. Soc. 1 (1935).

[6] R. J. Plemmons, Generalized inverses of Boolean relation matrices, SIAM J. Appl. Math. 20 (1971), 426-433.

7] P. S. S. N. V. Prasada Rao and K. P. S. Bhaskara Rao, On generalized inverses of Boolean matrices, Linear Algebra and Appl. 11 (1975), no. 2, 135-153.

[8] D. E. Rutherford, Inverses of Boolean matrices, Proc. Glasgow Math. Assoc. 6 (1963), 49-53.

[9] S. Z. Song, Linear operators that preserve column rank of Boolean matrices, Proc. Amer. Math. Soc. 119 (1993), no. 4, 1085-1088.

[10] S. Z. Song and K. T. Kang, Linear maps that preserve commuting pairs of matrices over general Boolean algebra, J. Korean Math. Soc. 43 (2006), no. 1, 77-86.

SEOK-Zun SONG

Department of Mathematics

JEJU NATional UNIVERSity

JEJU 690-756, KOREA

E-mail address: szsong@cheju.ac.kr

KYUNG-TAE KANG

Department of Mathematics

Jeju National University

JEJU 690-756, KoreA

E-mail address: kangkt@cheju.ac.kr

Mun-Hwan Kang

Department of Mathematics

JeJu NATional University

JEJU 690-756, KorEA

E-mail address: gudok@kornet.net 